\title{
SURFACE ENHANCED RAMAN SPECTROSCOPY OF HALIDE IONS ON COLLOIDAL SILVER: SIMULATION OF SILVER-HALIDE ION SPECTRA
}

\author{
Keith D. SHAW, Robin L. GARRELL and S. KRIMM \\ Department of Physics and Macromolecular Research Center, The University of Michigan, Ann \\ Arbor, Michigan 48109, USA
}

Received 15 June 1982; accepted for publication 15 November 1982

\begin{abstract}
The surface enhanced Raman spectra of halide ions adsorbed on colloidal silver surfaces can be explained by an interacting dipole model of a discrete adsorbate superlattice. Both (100) and (111) surfaces have been modeled, and simulated spectra for a range of adsorbate coverage fractions and sol particle facet sizes give good agreement with experimental spectra. The model is useful for assessing the nature and extent of adsorbate coverage at the aqueous-metal interface.
\end{abstract}

\section{Introduction}

Surface enhanced Raman spectroscopy (SERS) is a recently developed technique for studying molecules and ions adsorbed on metallic surfaces. While many studies have concentrated on the characteristics of the adsorbates, we have chosen to study properties of the surface itself. We recently reported SERS of halide ions on $\mathrm{Ag}$ sols [1] and have now presented a more detailed study of this system [2]. Although Nichols and Hexter [3] (NH) predicted that the $\mathrm{Ag}-\mathrm{X}^{-}$stretching frequency observed in electrochemical cells is shifted continuously by an appropriate coverage fraction of the total transition-dipole-transition-dipole interaction, we have shown $[1,2]$ that the observed bands in fact are comprised of discrete components. This fine structure is expected [1], since the net field of neighboring dipoles is not a continuous function of the coverage fraction, but in fact varies discretely with specific local arrangements of halide ions on the surface.

In this paper we present the details of our calculations. We have synthesized Raman spectra of the $\mathrm{Ag}-\mathrm{X}^{-}$stretching mode by modeling the dipole-dipole interactions of discrete adsorbate (100) and (111) superlattices. By varying the coverage fraction, both on very large as well as small facet-sized surfaces we obtain simulated spectra that are in good agreement with observed Raman bands of $\mathrm{Br}^{-}$and $\mathrm{Cl}^{-}$adsorbed on colloidal silver surfaces [1,2]. 


\section{Theory}

\subsection{Assumptions}

The model that we have used is based on the following assumptions. Since $\mathrm{Ag}$ is a face-centered cubic crystal, representative high atom density surfaces for adsorbates will be (100) planes (with a square lattice) and (111) planes (with a hexagonal lattice), each having a nearest neighbor $\mathrm{Ag}-\mathrm{Ag}$ spacing of $2.889 \AA$. We consider only commensurate superlattices of adsorbed ions, 4-coordinated to Ag on (100) planes and 3-coordinated on (111) planes. Since halide ions are charged, we assume that there is no tendency toward adsorbate aggregation (island formation). The cation has been shown [2] not to influence the $\mathrm{Ag}-\mathrm{X}^{-}$frequency, so we have treated the system only in terms of the halide ion interaction with the Ag surface. And dipole-dipole interactions between different adsorbed halide ions can be neglected since their characteristic $\mathrm{Ag}-\mathrm{X}^{-}$frequencies are significantly different.

We assume that every superlattice site is equally available. However, this does not imply, as has been the case in previous calculations [4-6], that only a single superlattice need be considered for all partial coverages. The halide ions studied are too large $\left(r\left(\mathrm{Cl}^{-}\right)=1.81 \AA, r\left(\mathrm{Br}^{-}\right)=1.96 \AA\right)$ to occupy adjacent superlattice sites, and therefore some sites will be excluded. But at less than maximum coverage, ions can be placed on Ag-coordinated sites that do not belong to a single regular superlattice, and the number of these arrangements is much larger than if ions are constrained to sites of a regular superlattice. In our simulation we have avoided this constraint, and have therefore sampled a larger variety of surface structures than was the case in previous models.

\subsection{Dipole sums}

We use the formulation of $\mathrm{NH}$ to calculate the frequency of the $\mathrm{Ag}-\mathrm{X}^{-}$ stretching mode interacting with the dipole field of neighboring adsorbates $\left(\mathrm{X}^{-}\right)$, namely

$\nu=\left[\nu_{0}+\frac{S(\chi)}{1+\alpha S(\chi)}\left(\frac{\partial \mu}{\partial q}\right)^{2}\right]^{1 / 2}$.

In this equation $\nu_{0}$ is the vibrational frequency of an isolated halide ion on the silver surface, estimated by NH from AgX crystal data and image effects; $S(\chi)$ is the value of the dipole sum of all adsorbate dipoles for a given coverage fraction $\chi ; \alpha$ is the polarizability of the halide ion real-plus-image system, and is twice the value of the electronic polarizability of an isolated halide ion; and $(\partial \mu / \partial q)$ is the dipole moment derivative of the $\mathrm{Ag}-\mathrm{X}^{-}$stretching mode. This derivative is very difficult to determine, so we have parametrized it, letting $\beta=(\partial \mu / \partial q)^{2}$. 
In order to discuss fractional coverage, a clear definition of full coverage is needed. We choose to define full coverage as the maximum density of halide ions that form a commensurate superlattice on a particular silver surface. The halide ions studied are too large to occupy all silver lattice sites. For the (100) face, the highest coverage configuration is a $c(2 \times 2)$ square superlattice of effective lattice constant $4.086 \AA$, where each adsorbed halide ion interacts with four silver surface atoms. The highest coverage configuration for a (111) face is a $(\sqrt{3} \times \sqrt{3}) \mathrm{R} 30^{\circ}$ hexagonal superlattice of effective lattice constant $5.004 \AA$, where each adsorbed halide ion interacts with three silver surface atoms. The Wood notation [7] is used, and drawings of these structures are given in fig. 1 .

The coverage fraction $x$ is therefore defined as the ratio of sites filled compared with the maximum number of allowed sites (e.g. a $\mathrm{c}(2 \times 2)$ superlattice).

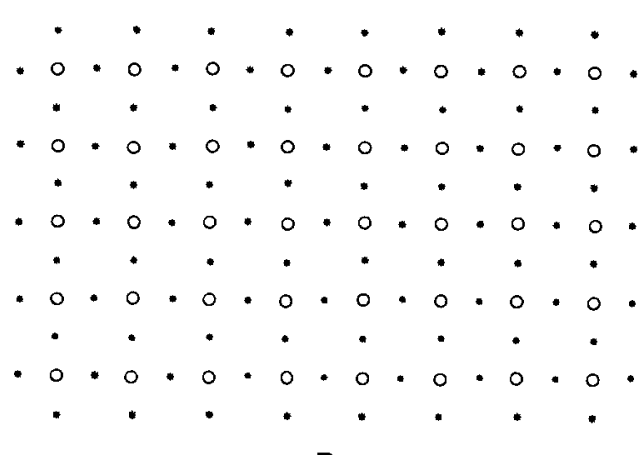

a

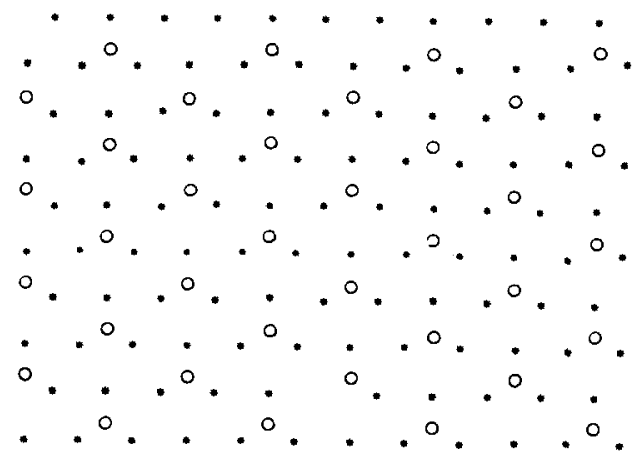

b

Fig. 1. Maximum coverage superlattice structures on (a) (100) and (b) (111) surfaces. Stars denote silver lattice sites, circles adsorbed ions. 
The dipole sum may be expressed as

$$
S(\chi)=\sum_{i j} n_{i j} \frac{1}{R_{i j}^{3}}
$$

where $i, j$ are the indices denoting surface position, $n_{i j}=1(0)$ indicating that the site at location $R_{i j}$ is filled (empty). Even though dipolar interactions are non-negligible at large distances, nearby neighbors dominate the total dipole sum. A vacancy of one near neighbor substantially reduces the value of the dipole sum, whereas a vacancy at a distant site has a much smaller effect. Moreover, the specific arrangement of nearby dipoles is much more important than the arrangement of distant ones.

To sum all the dipolar interactions on a partially filled infinite lattice would be a monumental task indeed! Outside some chosen vicinity radius $\left(R_{0}\right)$, where the discrete arrangement of dipoles is not important, we calculate the contributions of the remaining dipoles as a fraction of the remaining full coverage dipole sum. That is,

$$
\begin{aligned}
S(\chi) & =\sum_{i j} n_{i j} \frac{1}{R_{i j}^{3}}=\sum_{R_{i j} \leq R_{0}} n_{i j} \frac{1}{R_{i j}^{3}}+\sum_{R_{i j}>R_{0}} n_{i j} \frac{1}{R_{i j}^{3}} \\
& \simeq \sum_{R_{i j} \leq R_{0}} n_{i j} \frac{1}{R_{i j}^{3}}+\chi \sum_{R_{i j}>R_{0}} \frac{1}{R_{i j}^{3}} .
\end{aligned}
$$

For full coverage of a $\mathrm{c}(2 \times 2)$ superlattice

$$
S(1.0)=\sum_{i j} \frac{1}{R_{i j}^{3}}=\sum_{R_{i j} \leq R_{0}} \frac{1}{R_{i j}^{3}}+\sum_{R_{i j}>R_{0}} \frac{1}{R_{i j}^{3}},
$$

and

$$
\sum_{R_{i j}>R_{0}} \frac{1}{R_{i j}^{3}}=S(1.0)-\sum_{R_{i j} \leq R_{0}} \frac{1}{R_{i j}^{3}},
$$

therefore,

$$
S(\chi) \simeq \sum_{R_{i j} \leq R_{0}} n_{i j} \frac{1}{R_{i j}^{3}}+\chi\left(S(1.0)-\sum_{R_{i j} \leq R_{0}} \frac{1}{R_{i j}^{3}}\right) .
$$

Using the method of DeWette and Schacher [8], the two-dimensional infinite lattice sum is found to be $S(1.0)=9.0336 / A_{0}^{3}$ where $A_{0}$ is the lattice constant. Since we can also calculate the dipole sum for any particular superlattice inside the vicinity radius $R_{0}$, a value of $S(\chi)$ for any specific arrangement of adsorbed ions can be obtained. 


\subsection{Computer modeling}

\subsubsection{Infinite lattice calculation}

We begin with a $2 \times 2$ point array as the analogue of a (100) silver surface. To each array element we assign a random number of domain $(0,1)$. Then for any given coverage fraction $\chi$, array $(n, m) \leq \chi$ means the site at position $(n, m)$ is considered filled, and array $(n, m)>\chi$ means the site is empty. This gives a random mathematical array which simulates a partially filled lattice. If at each "filled" array element a poll is taken of neighboring site occupancy, a value of the dipole sum can be obtained and the resultant frequency calculated. By stepping through all filled array sites, a set of vibrational frequencies is produced. If we assume that all filled sites contribute equal SERS intensity, a histogram of this frequency distribution (occurrence versus frequency) yields a simulated Raman spectrum. Typically an array size was chosen such that 1000 to 10,000 such calculations were used to create a single spectrum. We note that for a fixed array size, lower coverage results in a lower amplitude histogram, a good representation of real coverage effects.

The adsorbed halide ions are, however, too large to occupy every silver lattice site. To represent this in the computer model, potential sites are forced empty (i.e. array $(n, m)$ is set equal to 1.0$)$ within the ionic diameter of an occupied site (i.e. one whose array $(n, m) \leq \chi)$. Certain sites are now automatically excluded from the dipole sum. Outside the vicinity radius we model the remaining array as a partially filled $c(2 \times 2)$ superlattice.

The only special consideration for a hexagonal array (to simulate the (111) surface) is to correctly define the distance between two array elements. For a (100) square lattice,

$R_{100}\left(\left(n_{i}, m_{i}\right) ;\left(n_{j}, m_{j}\right)\right)=\left[\left(n_{i}-n_{j}\right)^{2}+\left(m_{i}-m_{j}\right)^{2}\right]^{1 / 2}$,

but for a (111) hexagonal lattice

$R_{111}\left(\left(n_{i}, m_{i}\right) ;\left(n_{j}, m_{j}\right)\right)=\left[\left(n_{i}-n_{j}+\frac{1}{2}\left(m_{i}-m_{j}\right)\right)^{2}+\frac{3}{4}\left(m_{i}-m_{j}\right)^{2}\right]^{1 / 2}$.

Of course, outside the vicinity radius a $(\sqrt{3} \times \sqrt{3}) \mathrm{R} 30^{\circ}$ superlattice is used.

Even with relatively large statistics, the histograms appear somewhat "grainy". To smooth the spectra we have replaced each histogram element with a $1.5 \mathrm{~cm}^{-1}$ half-width Gaussian of the same integrated intensity.

We have also modeled surface defects in the silver lattice, such as point, slip plane, and screw dislocations. The effects of such surface defects on simulated Raman spectra are under investigation.

\subsubsection{Finite surface calculations}

While the calculation on an infinite lattice is informative in giving the general band contours and mean vibrational frequencies that result for given 
coverage fractions, this calculation is hardly realistic for sol particles whose facets have been determined by electron microscopy to be only $12-50 \AA$ in radius [2]. Since the number of sites on these facets is not prohibitive from a computational standpoint, we can explicitly sum all dipoles on the facet. The computer array simultaing the silver surface is partitioned into circular domains to represent the facets. Within a domain only a filled sites contribute to the dipole sum. A sufficient number of these domains are used to give statistics similar to those obtained in the infinite lattice calculation. Changing the domain radius simulates the effect on the Raman spectrum of finite facet size.

\subsubsection{Parameters}

The parameters used in calculating a simulated Raman spectrum are $\nu_{0}, \alpha$, $\beta, \chi$, and the vinicity (or facet) radius $R_{0}$. We assume for simplicity that $\nu_{0}$ (the isolated vibrational frequency) and $\beta$ (the dipole moment derivative parameter) are independent of the underlying silver lattice type and of surface coverage $\chi$.

Nichols and Hexter's [3] values for $\nu_{0}\left(159 \mathrm{~cm}^{-1}\right.$ for $\mathrm{Cl}^{-}, 100 \mathrm{~cm}^{-1}$ for $\left.\mathrm{Br}^{-}\right)$and $\alpha\left(5.70 \AA^{3}\right.$ for $\mathrm{Cl}^{-}, 8.18 \AA^{3}$ for $\left.\mathrm{Br}^{-}\right)$have been used. The parameters $\nu_{0}$ and $\alpha$, and the dipole sum $S(\chi)$ for a given coverage uniquely determine the band contour, while $\beta$ scales the frequencies with respect to $\nu_{0}$. We have chosen $\beta$ so that the calculated frequencies of major band components match experimental components in bands having similar profiles (i.e. similar coverage). Our values of $\beta$ are $6.77 \times 10^{-19} \mathrm{~cm}^{-1}$ for $\mathrm{Cl}^{-}$and $5.80 \times 10^{-19} \mathrm{~cm}^{-1}$ for $\mathrm{Br}^{-}$.

\section{Results and discussions}

\subsection{Generated spectra}

The infinite lattice calculations were performed with a vicinity radius of 4 lattice constants (about $12 \AA$ ), since we found that the resulting spectra do not differ substantially from those obtained using vicinity radii of up to 14 lattice constants (about $40 \AA$ ). The array size was fixed at 5000 array elements for all calculations.

For the (100) face, fig. 2 shows the dependence of band profile on coverage fraction. The major features of the spectrum arise from nearest neighbor vacancies; one can in fact label the peaks by the number of missing nearest neighbors. In fig. 2c (coverage fraction of 0.55 ), the peaks at 260, 250, 241, 232, and $217 \mathrm{~cm}^{-1}$ correspond to $0,1,2,3$, and 4 vacant nearest neighbor sites, respectively. For 0.50 coverage, one would expect two missing nearest neighbors to be the most common proximal configuration, and indeed the highest peak at $242 \mathrm{~cm}^{-1}$ agrees with this nicely. For lower coverage spectra this labeling becomes inadequate, since effects of second nearest neighbors become 

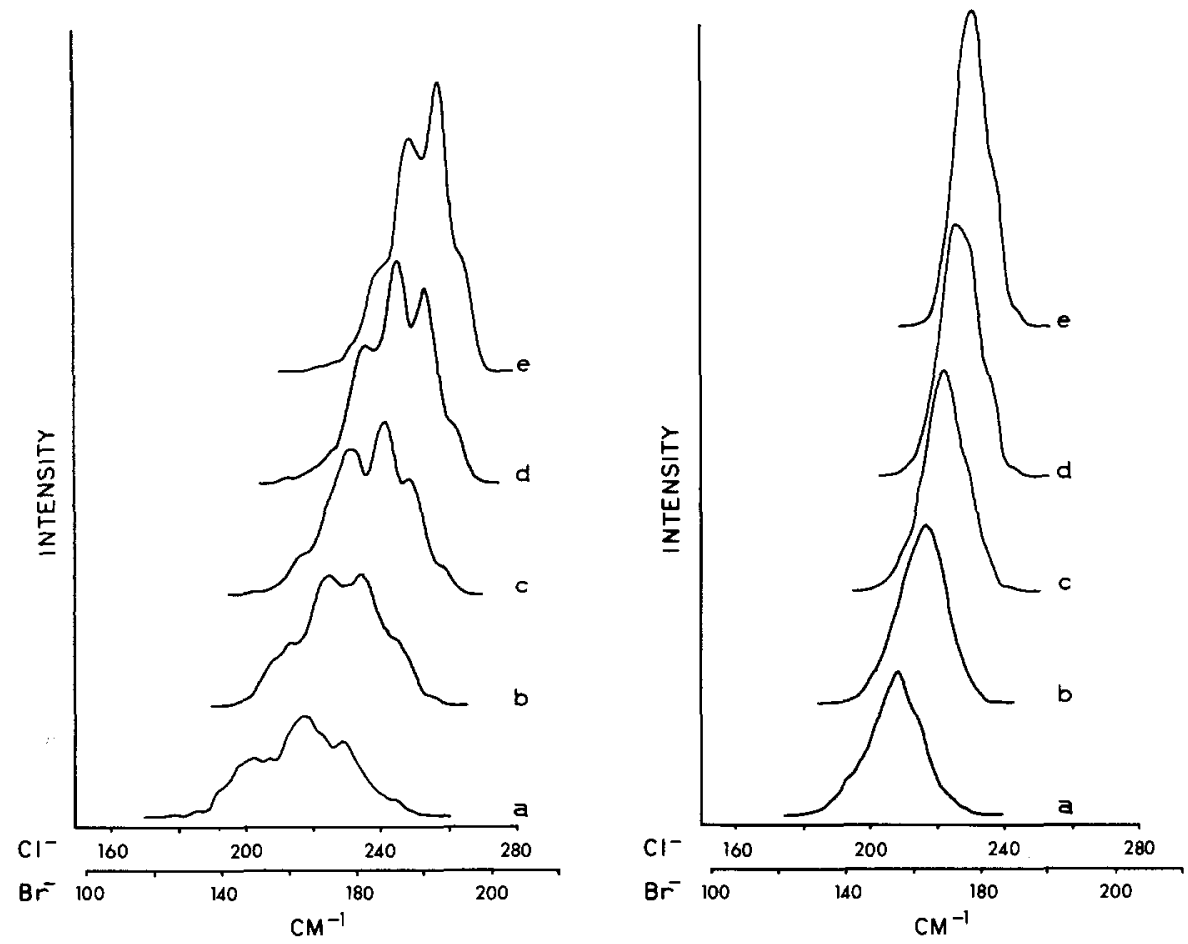

Fig. 2. Simulated halide ion spectra as a function of adsorbate coverage fraction, for an infinite (100) surface: (a) 0.40 coverage; (b) 0.48 ; (c) 0.55 ; (d) 0.62 ; (e) 0.70 .

Fig. 3. Coverage fraction effects on simulated spectra for adsorption on an infinite (111) surface: (a) 0.48 coverage; (b) 0.57 ; (c) 0.64 ; (d) 0.69 ; (e) 0.74 .

relatively more important (as shown by the increased complexity of the band profile of fig. 2a). Together with the general trend of lower mean frequency with decreasing coverage, an increase in bandwidth is predicted by the model. These predictions are correlated with experimental evidence in a later section of this paper.

Infinite lattice calculations for the (111) face, given in fig. 3, show remarkably featureless band contours, substantially narrower bandwidths, and lower mean frequencies as compared to the $(100)$ simulations of the same coverage fractions. Since the sol surface consists of different types of facets, the experimental band shapes resulting from adsorption on (111) surfaces may be obscured by the broader more complex (100) profiles. There are no Raman spectra of halide ions on pure (100) or (111) silver surfaces, and therefore we cannot verify the (111) surface predictions. Recent IR studies [9] of adsorbed $\mathrm{CO}$ on carefully prepared $\mathrm{Ni}$ surfaces, however, show a lower $\mathrm{Ni}-\mathrm{CO}$ vibra- 
tional frequency on the (111) surface as compared to the (100) surface. This would imply a lower $\nu_{0}$ in our case, even though we assumed $\nu_{0}$ to be surface-independent. If $\nu_{0}$ were indeed lower, the entire (111) spectrum would be shifted to a substantially lower frequency region; but our SERS scans down to $50 \mathrm{~cm}^{-1}$ show no evidence for this phenomenon. Hence either adsorption on (111) surfaces does not occur, or the (111) band profile is obscured by the (100) contributions. Quite possibly the presence of solvent and of counterions in our experimental system cause $\nu_{0}$ to shift only slightly compared to the significant shifts observed for adsorbed gases under ultrahigh vacuum conditions.

Figs. 4, 5, and 6 show the theoretical spectra for various (100) facet sizes with coverage fractions of $0.7,0.55$, and 0.4 respectively. For any given coverage fraction, decreasing the facet size shifts the mean frequency downward (fewer dipoles being available) and increases the overall bandwidth. Also, new components appear on the low frequency side of the band. Adsorbed ions
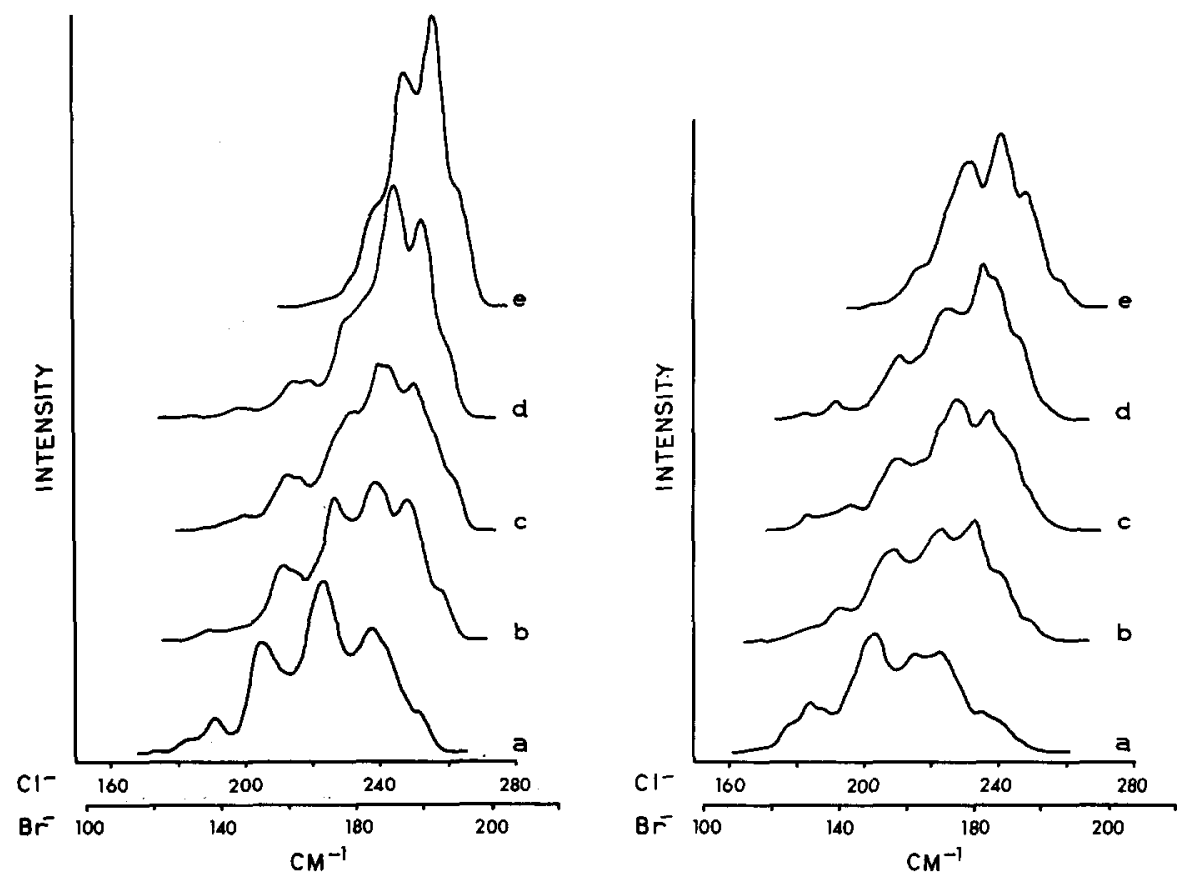

Fig. 4. Effect of facet size on simulated spectra for 0.70 coverage fraction on a (100) surface. Facet radii: (a) $12 \AA$; (b) $20 \AA$; (c) $29 \AA$; (d) $40 \AA$; (e) infinite lattice.

Fig. 5. Effect of facet size on simulated spectra for 0.55 coverage fraction on a (100) surface. Facet radii: (a) $12 \AA$; (b) $20 \AA$; (c) $29 \dot{A}$; (d) $40^{\circ}$; (e) infinite lattice. 
near the edge of a facet have, in general, fewer close neighbors than those near the facet center, and therefore give rise to significantly lower frequencies. The nearer the adsorbed ion is to the edge, the larger the change in the dipole sum, and the greater are the contributions of the low frequency components.

For the (111) surface, fig. 7 shows the facet size effect for a 0.73 coverage fraction. Since the infinite lattice calculation (fig. 7e) gives a virtually featureless band, the distinct features of the facet calculations are due entirely to edge effects. One can almost label the major features by the number of lattice units from the edge. In fig. $7 \mathrm{a}$, bands at 195,209 and $215 \mathrm{~cm}^{-1}$ correspond to dipoles 0,1 , and 2 units from the edge, respectively.

In all cases spectra have been calculated for coverage fractions of 0.1 to 0.9 , but we have included here only those from 0.4 and 0.7 . Virtually all our recorded experimental spectra appear to correspond to this narrower coverage range. Lower coverages may give spectra too weak to observe, or may be
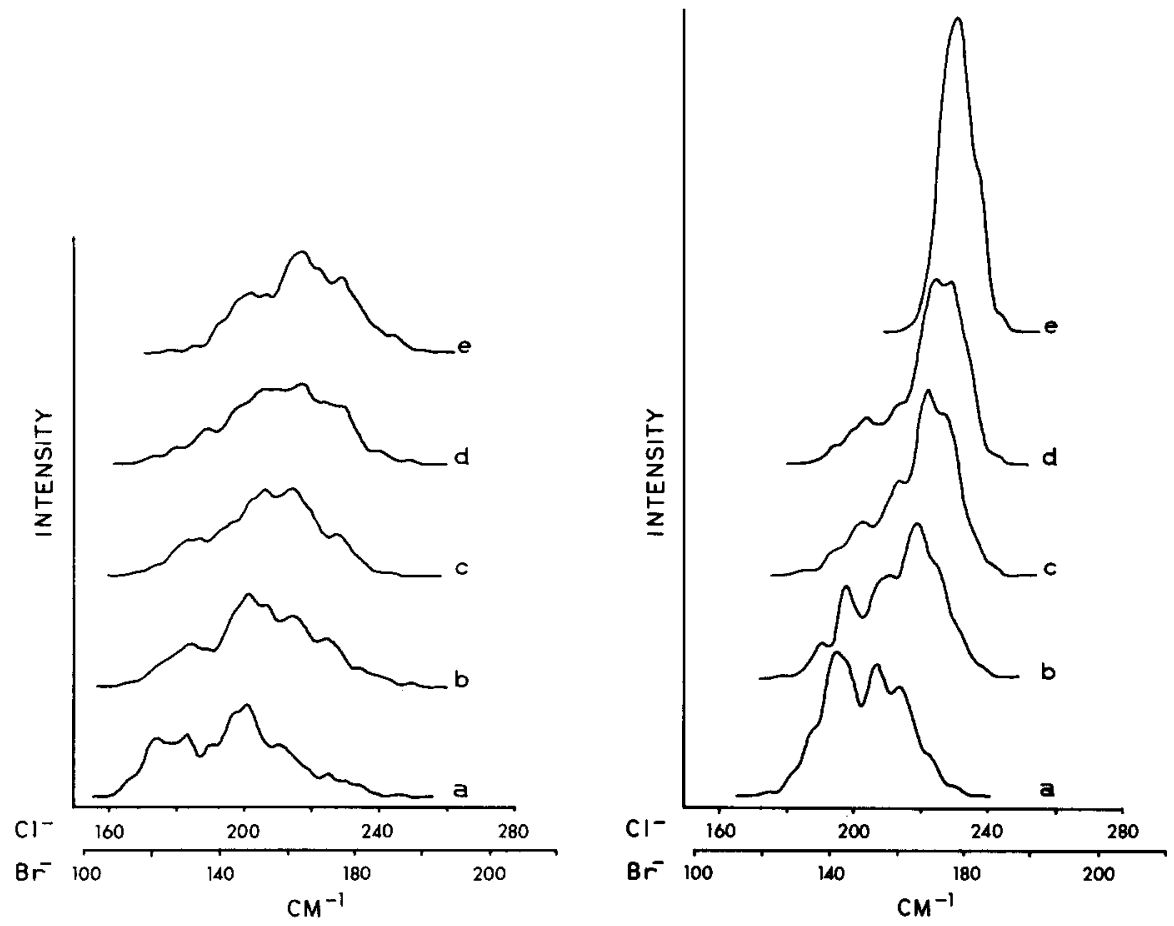

Fig. 6. Effect of facet size on simulated spectra for 0.40 coverage fraction on a (100) surface. Facet radii: (a) $12 \AA$; (b) $20 \AA$; (c) $29 \AA$; (d) $40 \AA$; (e) infinite lattice.

Fig. 7. Facet size effects on simulated spectra for 0.73 coverage fraction on a (111) surface. Facet radii: (a) $12 \AA$; (b) $20 \AA$; (c) $29 \AA$; (d) $40 \AA$; (e) infinite lattice. 
disallowed because of the nature of the adsorption isotherm (see preceding paper). The absence of higher adsorbate coverage fractions could be a consequence of charge repulsion. Since the silver sol particle is electrostatically

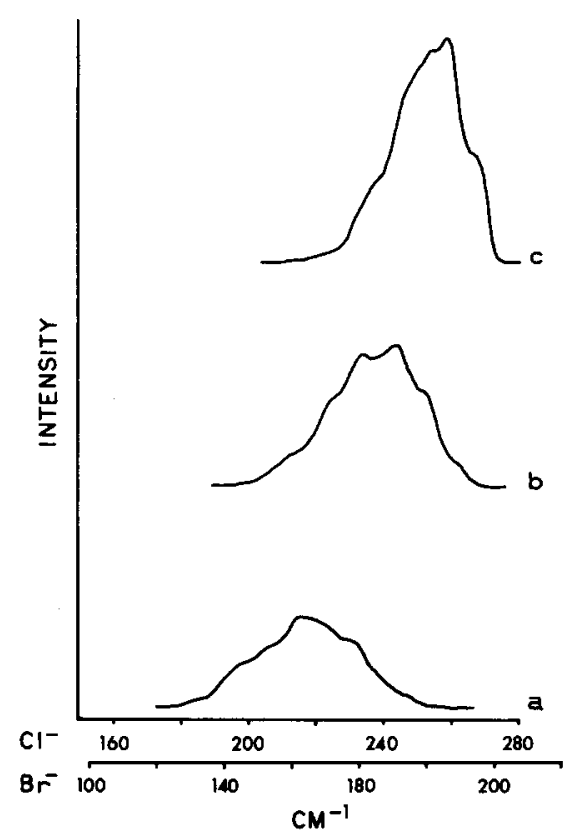

Fig. 8. Simulated spectra for an average of coverage fractions on an infinite (100) surface: (a) 0.35 , 0.39 , and 0.44 coverage fractions; (b) $0.48,0.55$, and 0.62 ; (c) $0.62,0.69$, and 0.77 . (a), (b), and (c) simulate coverage fractions of roughly $0.39,0.55$, and 0.69 respectively.

isolated, the adsorption of many negatively charged halide ions may eventually inhibit additional adsorption.

For calculated high coverage fractions on the (100) surface the band components often appear more distinct than is observed experimentally. We have done calculations in which spectra for a small range of coverage fractions were averaged to represent a slight variance in coverage fraction on large lattices. For example, averaging coverage fractions of $0.62,0.69$, and 0.77 , as is shown in fig. 8c, almost completely removes the distinctness of the band components, while the mean frequency, bandwidth, and shoulder frequency positions of the original 0.69 coverage spectrum are retained. Such slight variability in coverage fraction may be the reason for the less distinctive band contours observed experimentally. 


\subsection{Comparison of theoretical with observed spectra}

While a specific band-by-band comparison of the calculated with the observed spectra is not yet feasible, we feel we can at least explain the main features of the observed halide ion spectra. We discuss below the $\mathrm{Br}^{-}$and $\mathrm{Cl}^{-}$ spectra presented in the previous paper (ref. [2], figs. $3 a-3 e$, referred to below by just the figure number).

Though the calculated single fractional coverage spectra for (100) faces (faceted or infinite lattice) are more featured than the observed full coverage $\mathrm{Br}^{-}$and $\mathrm{Cl}^{-}$spectra, $3 \mathrm{a}$ and $3 \mathrm{e}$, an average of fractional coverages can give spectra strikingly similar in mean frequency, bandwidth and features to the observed spectra. The $\mathrm{Br}^{-}$spectrum $3 \mathrm{a}$ can be described by an average of coverage fractions $0.35-0.44$, as in fig. $8 \mathrm{a}$. Since $\mathrm{Br}^{-}(d=3.92 \AA)$ is almost large enough to prevent adsorption on next nearest sites (at $\sqrt{2} a_{0}=4.092 \AA$ ), high $\mathrm{Br}^{-}$coverage is not expected. We have never observed a mean frequency for $\mathrm{Br}^{-}$higher than that shown in $3 \mathrm{a}$, which suggests that coverage fractions greater than 0.5 on (100) surfaces are unlikely. Similarly, spectrum $3 \mathrm{~b}$ can be described by $0.4-0.48$ coverage, and so on.

The facet calculations are of course more realistic: the experimental spectrum 3a, for example, can be described as an average of $0.4-0.5$ coverage on roughly $30 \AA$ facets, while spectrum $3 \mathrm{c}$ corresponds to 0.5 coverage on $20 \AA$ facets. In general, a decrease in the observed mean frequency can be a consequence of either a decrease in coverage fraction or in facet size, the latter also resulting in an overall band broadening and in the development of relatively conspicuous features on the low frequency side of the band. This effect is more clearly in evidence in the $\mathrm{Cl}^{-}$spectra (ref. [2], figs. 3e-3h).

Chloride spectrum $3 \mathrm{e}$ is well matched by the average of $0.62,0.69$, and 0.77 coverage fractions on (100), as shown in fig. 8c. It would then follow that $\mathrm{Cl}^{-}$ partial coverage spectra should correlate with reduced coverage or reduced facet size. The position and breadth of observed spectrum $3 \mathrm{~h}$ corresponds roughly to the coverage range $0.48,0.55,0.62$ shown in fig. $8 \mathrm{~b}$. But in fact, since the observed spectrum is more highly featured, it would be more accurately described by 0.55 coverage on an infinite lattice (fig. 5e) or large (40 A) facets (fig. 5d).

The bands in the $\mathrm{Cl}^{-}$spectra from aggregated sols (3f and $3 \mathrm{~g}$ ) are much broader than those from unaggregated sols ( $3 \mathrm{e}$ and $3 \mathrm{~h}$ ). Spectra of such large bandwidth can result from adsorption on facets of a range of sizes. The low frequency region would be expected to show the distinct features characteristic of adsorption on small facets. Thus, $\mathrm{Cl}^{-}$spectra $3 \mathrm{~g}$ and $3 \mathrm{~h}$ could be explained as resulting from low-to-moderate coverage on large facets (approx. $40 \AA$ radius) along with similar coverage on smaller facets.

These results lead to two distinct physical interpretations of the aggregation phenomenon. One is that aggregation somehow decreases the average size of 
the facets available for adsorption. The other possibility is that in aggregated sols adsorption occurs both on the larger facets (on which it adsorbs in unaggregated sols) and additionally on small facets. The additional adsorption on small facets might occur because of a decreased availability of large facet adsorption sites, or because of some induced change in the small facet sites that somehow facilitates adsorption. Note that the $\mathrm{Br}^{-}$spectra from aggregated sols do not show the enlarged bandwidth observed for the $\mathrm{Cl}^{-}$spectra. Any description of the aggregation process must account for this observation as well.

In general, band shapes generated for (111) lattices (figs. 3 and 7) are too narrow and insufficiently featured to correspond directly to any of the observed $\mathrm{Br}^{-}$spectra, $3 \mathrm{a}-3 \mathrm{~d}$, or $\mathrm{Cl}^{-}$spectra, $3 \mathrm{e}-3 \mathrm{~h}$. The (111) component may simply be obscured by the broader, more featured (100) profile in the observed spectra. The explicit contribution from (111) faces cannot be described at this time, but the overall features of the adsorbed halide ion spectra can be explained by adsorption primarily on (100) faces. This is also consistent with the notion that the adsorbed ions prefer sites of higher coordination number [10]. Hence both $\mathrm{Br}^{-}$and $\mathrm{Cl}^{-}$are expected to prefer (100) 4-coordinate sites to (111) 3-coordinate sites.

\section{Conclusions}

The general agreement between our simulated spectra and the observed spectra of halide ions adsorbed on colloidal silver indicates that the essential experimental features can be explained by an interacting transition dipole model of the discrete adsorbate superlattice, with the finite substrate lattice being accounted for explicitly. This, of course, does not prove that other phenomena may not also be involved, such as through-metal effects. However, the fact that the fine structure on the $\mathrm{Ag}-\mathrm{X}^{-}$bands, together with its variation with surface coverage, can be understood in terms of interacting transition dipoles means that this mechanism must be given serious consideration as the underlying basis for the observations. Additional evidence would be provided from studies on well-defined homogeneous silver surfaces of known facet size, since it is not possible at present to characterize the colloidal $\mathrm{Ag}$ surface in such detail. Our model nevertheless offers a tool for assessing the extent and nature of adsorbate coverage at the aqueous-metal interface, a system to which most conventional surface analytical techniques cannot be applied.

\section{Acknowledgements}

This work was supported by National Science Foundation grants DMR 78-00753 and PCM 79-21652, and by the Macromoleculer Research Center (R.L.G.). 


\section{References}

[1] R.L. Garrell, K.D. Shaw and S. Krimm, J. Chem. Phys. 75 (1981) 4155.

[2] R.L. Garrell, K.D. Shaw and S. Krimm, Surface Sci. 124 (1982) 613.

[3] H. Nichols and R.M. Hexter, J. Chem. Phys. 74 (1981) 2059.

[4] A. Crossby and D.A. King, Surface Sci. 68 (1977) 528.

[5] G.D. Mahan and A.A. Lucas, J. Chem. Phys. 68 (1978) 1344.

[6] H. Nichols and R.M. Hexter, J. Chem. Phys. 76 (1982) 5595.

[7] E.A. Wood, J. Appl. Phys. 35 (1964) 1306.

[8] E.W. DeWette and G.E. Schacher, Phys. Rev. A137 (1965) 78.

[9] J.C. Bertolini and B. Tardy, Surface Sci. 102 (1981) 131.

[10] G.A. Somorjai, Chemistry in Two Dimensions: Surfaces (Cornell University Press, Ithaca, NY, 1981) p. 270. 